\title{
Automated Analysis of EDS Maps
}

\author{
Christian Lang ${ }^{1}$ and Matthew Hiscock ${ }^{1}$
}

1. Oxford Instruments Nanoanalysis, High Wycombe, United Kingdom.

With increases in EDS detector speed and the use of multiple EDS detectors on one SEM, EDS is rapidly moving from being a purely spectroscopic technique to becoming an imaging technique. As the beam is rastered over the sample and an X-ray spectrum is recorded at every pixel an X-ray map is built up that can qualitatively indicate the presence of different phases in the sample (Figure 1b). If the spectra acquired during X-ray mapping are live-time corrected, they can be processed and quantified like any other spectrum. This enables the reconstruction and quantification of spectra from features of interest in maps. So far this reconstruction has generally been a manual process where the user defines the area from which to reconstruct and then examines the spectrum and draws conclusions about the presence of certain phases. Here we discuss the combination of image processing and classification with EDS mapping tools to create a virtual sample from which morphological and chemical features can be automatically determined.

We have previously presented a solution to acquire and stitch EDS maps and electron images over large areas automatically [1]. The main use to acquire the electron image together with the map was to use the electron image as a reference for drift correction and automated alignment. However, as both the electron image and the EDS map are spatially registered, it is possible to use image processing on the electron image to identify features of interest and then extract compositional information from the maps automatically. This process is shown in Figure 1(a) where the backscattered electron (BSE) image clearly shows different phases in the sample. By thresholding the BSE image we identify features of interest, in this case different mineral phases in the sample. We can then use the regions identified by thresholding to extract the EDS spectra from the X-ray maps (figure 1(b)). The EDS spectra are quantified and by using a classification routine identical to approaches used in particle analysis [2] we can assign phases to the different regions which can be represented by different colours (figure 1(c)).

A similar result can be achieved by processing EDS maps directly and using an algorithm such as Aztec AutoPhaseMap to group pixels of similar composition. As we show in Figure 2, this achieves a similar result to the above process in terms of identifying phases of interest and does not rely on the presence of an electron image. However, there are advantages and disadvantages to this approach. First, in order to achieve a successful grouping of pixels, the statistics in each pixel need to be good enough to make a reliable decision on whether its phase is identical or different from any neighboring pixel. Even though the acquisition of EDS data is fast compared to several years ago, it still takes significantly longer to acquire an EDS map with good statistics compared to an electron image. In image processing terms we are dealing therefore with a noisier data set or require longer acquisition times. Also, depending on the electron beam energy used, the X-ray interaction volume may be considerably larger than that of the electron beam, leading to a degraded spatial resolution. However, using the X-ray data directly also has advantages. If two phases have very similar density it may not be possible to distinguish them by BSE contrast, whereas they may be easily distinguished from the maps. Also, identifying the correct phases from the BSE image relies on setting the correct threshold limits. This may require some a priori knowledge or investigation of the sample. If there are sufficient counts in each pixel of the map the AutoPhaseMap algorithm will identify regions of similar composition automatically and therefore not 
require any pre-knowledge or user intervention.

In summary, we show how approaches used in particle analysis such as thresholding and classification can be successfully applied to enable the automated analysis of X-ray maps and compared the results to purely mapping based algorithms such as AutoPhaseMap. Which approach is more successful depends on the type of sample and spatial resolution required from the analysis.

\section{References:}

[1] S Burgess et al, Microscopy and Microanalysis 20 (2014), p. 640.

[2] C Lang et al, Microscopy and Microanalysis 21 (2015), p. 337.
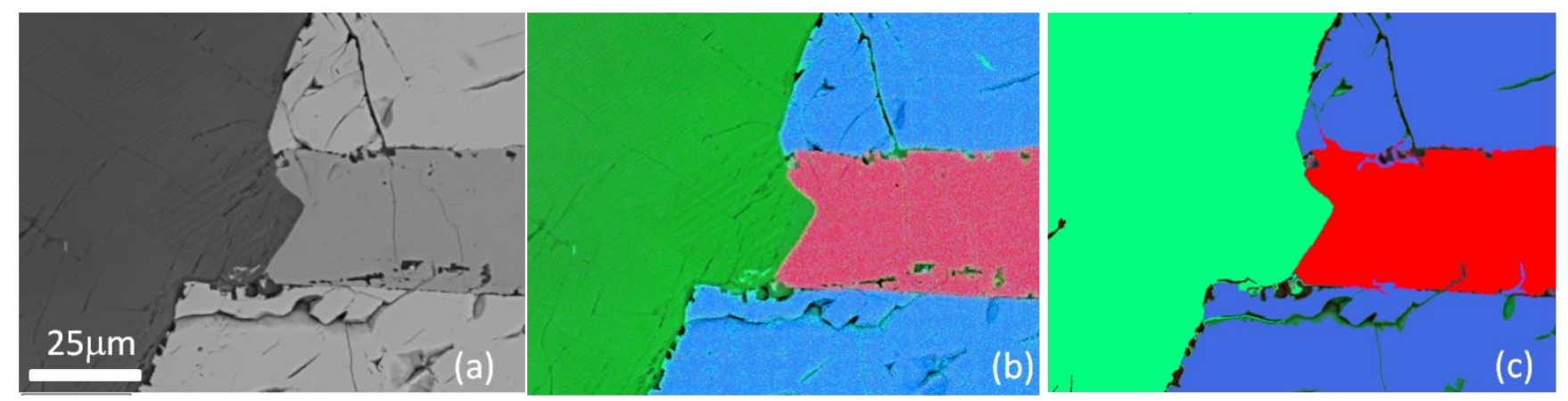

Figure 1. (a) BSE image showing different density of the minerals present in the sample. (b) EDS overlay map qualitatively showing different phases by overlaying different element maps (c) result of automated analysis of the X-ray map by reconstructing and classifying spectra from regions of similar contrast in the BSE image.

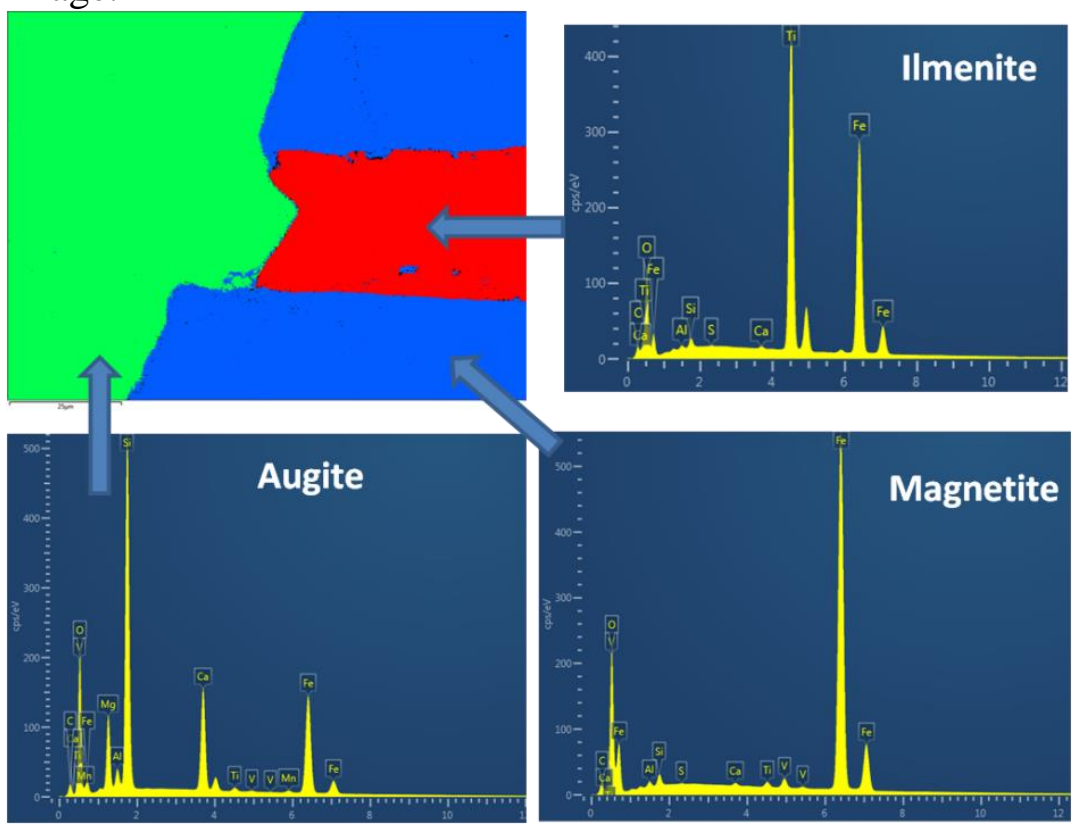

Figure 2. Automated analysis of the X-ray map by grouping pixels in the map using the AutoPhaseMap algorithm. The spectra shown are the sum spectra of pixels assigned to the same phase. 